Proceedings of the Edinburgh Mathematical Society (2005) 48, 1-19 (C)

DOI:10.1017/S0013091503000774 Printed in the United Kingdom

\title{
SOLVABILITY OF SINGULAR DIRICHLET BOUNDARY-VALUE PROBLEMS WITH GIVEN MAXIMAL VALUES FOR POSITIVE SOLUTIONS
}

\author{
RAVI P. AGARWAL ${ }^{1}$, DONAL O'REGAN ${ }^{2}$ AND SVATOSLAV STANĚK ${ }^{3}$ \\ ${ }^{1}$ Department of Mathematical Sciences, Florida Institute of Technology, \\ Melbourne, FL 32901-6975, USA (agarwal@fit.edu) \\ ${ }^{2}$ Department of Mathematics, National University of Ireland, \\ Galway, Ireland (donal.oregan@nuigalway.ie) \\ ${ }^{3}$ Department of Mathematical Analysis, Faculty of Science, Palacký University, \\ Tomkova 40, 77900 Olomouc, Czech Republic (stanek@risc.upol.cz)
}

(Received 9 September 2003)

\begin{abstract}
The singular boundary-value problem $\left(g\left(x^{\prime}(t)\right)\right)^{\prime}=\mu f\left(t, x(t), x^{\prime}(t)\right), x(0)=x(T)=0$ and $\max \{x(t): 0 \leqslant t \leqslant T\}=A$ is considered. Here $\mu$ is the parameter and the negative function $f(t, u, v)$ satisfying local Carathéodory conditions on $[0, T] \times(0, \infty) \times(\mathbb{R} \backslash\{0\})$ may be singular at the values $u=0$ and $v=0$ of the phase variables $u$ and $v$. The paper presents conditions which guarantee that for any $A>0$ there exists $\mu_{A}>0$ such that the above problem with $\mu=\mu_{A}$ has a positive solution on $(0, T)$. The proofs are based on the regularization and sequential techniques and use the Leray-Schauder degree and Vitali's convergence theorem.
\end{abstract}

Keywords: singular boundary-value problem; Dirichlet problem; existence; positive solution;

Leray-Schauder degree; Vitali's convergence theorem

2000 Mathematics subject classification: Primary 34B16; 34B18

\section{Introduction}

Let $T$ be a positive constant, $J=[0, T], \mathbb{R}_{+}=(0, \infty)$ and $\mathbb{R}_{0}=\mathbb{R} \backslash\{0\}$. We will consider the singular boundary-value problem (BVP)

$$
\begin{gathered}
\left(g\left(x^{\prime}(t)\right)\right)^{\prime}=\mu f\left(t, x(t), x^{\prime}(t)\right), \\
x(0)=0, \quad x(T)=0, \\
\max \{x(t): t \in J\}=A,
\end{gathered}
$$

where $g \in C^{0}(\mathbb{R} ; \mathbb{R})$ is an increasing and odd function, $f$ satisfies local Carathéodory conditions on $J \times \mathbb{R}_{+} \times \mathbb{R}_{0}\left(f \in \operatorname{Car}\left(J \times \mathbb{R}_{+} \times \mathbb{R}_{0} ; \mathbb{R}\right)\right), \mu \in \mathbb{R}$ is a parameter and $A \in \mathbb{R}_{+}$. The function $f(t, u, v)$ may be singular at the value $u=0$ and $v=0$ of the phase variables $u$ and $v$, respectively. 
Let $A \in \mathbb{R}_{+}$. We say that $x$ is a solution of $B V P(1.1)-(1.3)$ if

(i) $x \in C^{1}(J ; \mathbb{R}), g\left(x^{\prime}\right) \in \mathrm{AC}(J ; \mathbb{R})$ (that is, $g\left(x^{\prime}\right)$ is absolutely continuous on $\left.J\right), x>0$ on $(0, T)$;

(ii) $x$ satisfies the boundary conditions (1.2), (1.3); and

(iii) there exists $\mu_{A} \in \mathbb{R}$ such that $x$ satisfies (1.1) with $\mu=\mu_{A}$ for a.e. $t \in J$.

We recall that any solution of BVP (1.1)-(1.3) starts and finishes at the singular point of $f$ in the first phase variable and at an inner point of the interval $J$ 'passes through' the singular point of $f$ in the second phase variable.

The purpose of this paper is to give conditions on the functions $g$ and $f$ in (1.1) which guarantee the solvability of BVP (1.1)-(1.3) for each $A \in \mathbb{R}_{+}$. Problems of the above form have received very little attention in the literature (see $[\mathbf{3}]$ and references therein). In $[\mathbf{3}]$ the authors discussed the Lidstone BVP

$$
\begin{gathered}
(-1)^{n} x^{(2 n)}(t)=\mu h\left(x(t), x^{\prime}(t), \ldots, x^{(2 n-2)}(t)\right), \\
x^{(2 j)}(0)=x^{(2 j)}(T)=0, \quad 1 \leqslant j \leqslant n-1,
\end{gathered}
$$

together with the condition (1.3) and where $h$ may be singular at the zero value of all its phase variables.

We note that problem (1.1), (1.2) is a singular Dirichlet BVP depending on the parameter $\mu$, and so we are looking for a value of $\mu$ for which problem (1.1), (1.2) has a positive solution on $(0, T)$ satisfying (1.3). Under the assumption that problem (1.1), (1.2) has a unique positive solution for each $\mu$ from a subset of $\mathbb{R}$, the shooting method can be applied for solving the BVP (1.1)-(1.3). This procedure was used, for example, in [16] for a special form of the function $f$ and when $g(u) \equiv u$, and the authors consider signchanging solutions. In this paper the uniqueness of solutions to problem (1.1), (1.2) is not assumed. Another method, based on the implementation of parameters to differential equations, functional differential equations and their systems with additional conditions, has been employed, for instance, in $[\mathbf{6}, \mathbf{9}, \mathbf{1 1}, \mathbf{1 3}, \mathbf{1 7}-\mathbf{2 1}]$.

We observe that the singular Dirichlet problem (1.1), (1.2) with $\mu=1$ and $g(u) \equiv u$ in (1.1) and non-positive $f(t, u, v)$ which may be singular at the zero value of the phase variable $u$ and having positive solution on $(0, T)$ has been considered in the literature (see, for example, $[\mathbf{1}, \mathbf{2}, \mathbf{8}, \mathbf{1 2}, \mathbf{1 4}, \mathbf{1 5}, \mathbf{2 2 - 2 7}]$, and references therein). Here solutions were considered in the class $C^{0}(J) \cap C^{2}((0, T))$ or $C^{1}(J) \cap C^{2}((0, T))$ or $C^{0}(J) \cap A C_{\text {loc }}^{1}((0, T))$.

To prove existence results for BVP (1.1)-(1.3), we use the following procedure. First, we define a set of regular differential equations depending on $n \in \mathbb{N}$ and, using the LeraySchauder topological degree and the Borsuk antipodal theorem (see, for example, [7]), we show that a sequence of auxiliary regular BVPs of the type (1.1)-(1.3) has a sequence $\left\{x_{n}\right\}$ of solutions. Then, applying the Arzelà-Ascoli theorem we verify that a convergent subsequence can be selected from $\left\{x_{n}\right\}$. Finally, by Vitali's convergence theorem (see, for example, $[\mathbf{5}, \mathbf{1 0}])$, we prove that its limit is a solution of BVP (1.1)-(1.3). 
From now on,

$$
\|x\|=\max \{|x(t)|: t \in J\} \quad \text { and } \quad\|x\|_{\infty}=\operatorname{essmax}\{|x(t)|: t \in J\}
$$

stand for the norms in $C^{0}(J ; \mathbb{R})$ and $L_{\infty}(J ; \mathbb{R})$, respectively. For a measurable set $\mathcal{M} \subset \mathbb{R}$, $\mu(\mathcal{M})$ denotes the Lebesgue measure of $\mathcal{M}$.

In this paper we use the following assumptions.

$\left(\mathrm{H}_{1}\right) g \in C^{0}(\mathbb{R} ; \mathbb{R})$ is increasing, $g(-u)=-g(u)$ for $u \in \mathbb{R}, \lim _{u \rightarrow \infty} g(u)=\infty$, and there exists $\beta \in \mathbb{R}_{+}$such that

$$
g(u) \leqslant u^{\beta} \quad \text { for } u \in \mathbb{R}_{+} .
$$

$\left(\mathrm{H}_{2}\right) f \in \operatorname{Car}\left(J \times \mathbb{R}_{+} \times \mathbb{R}_{0} ; \mathbb{R}\right)$ and there exists $a \in \mathbb{R}_{+}$such that

$$
a \leqslant-f(t, u, v) \text { for a.e. } t \in J \text { and each }(u, v) \in \mathbb{R}_{+} \times \mathbb{R}_{0} .
$$

$\left(\mathrm{H}_{3}\right)$ For a.e. $t \in J$ and each $(u, v) \in \mathbb{R}_{+} \times \mathbb{R}_{0}$,

$$
-f(t, u, v) \leqslant \varphi(t)\left[h_{1}(u)+h_{2}(u)\right]\left[\omega_{1}(g(|v|))+\omega_{2}(g(|v|))\right],
$$

where $\varphi \in L_{\infty}\left(J ; \mathbb{R}_{+}\right), h_{1}, \omega_{1} \in C^{0}\left([0, \infty) ; \mathbb{R}_{+}\right)$are non-decreasing, $h_{2}, \omega_{2} \in$ $C^{0}\left(\mathbb{R}_{+} ; \mathbb{R}_{+}\right)$are non-increasing, and

$$
\begin{gathered}
\int_{0}^{1} h_{2}(s) \mathrm{d} s<\infty, \quad \int_{0}^{1} \omega_{2}(s) \mathrm{d} s<\infty \\
\int_{0}^{\infty} \frac{\sqrt[\beta]{s}}{\omega_{1}(s)} \mathrm{d} s=\infty
\end{gathered}
$$

Remark 1.1. Since $h_{2}, \omega_{2}: \mathbb{R}_{+} \rightarrow \mathbb{R}_{+}$are non-increasing, (1.5) implies that

$$
\int_{0}^{V} h_{2}(s) \mathrm{d} s<\infty, \quad \int_{0}^{V} \omega_{2}(s) \mathrm{d} s<\infty \quad \text { for each } V \in \mathbb{R}_{+} .
$$

\section{Auxiliary regular BVPs}

Throughout this section we assume that assumptions $\left(\mathrm{H}_{1}\right)-\left(\mathrm{H}_{3}\right)$ are satisfied. For each $n \in \mathbb{N}$, define $\chi_{n} \in C^{0}\left(\mathbb{R} ; \mathbb{R}_{+}\right)$and $f_{n} \in \operatorname{Car}\left(J \times \mathbb{R}^{2} ; \mathbb{R}\right)$ by

$$
\chi_{n}(u)= \begin{cases}u & \text { for } u \geqslant \frac{1}{n}, \\ \frac{1}{n} & \text { for } u<\frac{1}{n},\end{cases}
$$

and

$$
f_{n}(t, u, v)= \begin{cases}f\left(t, \chi_{n}(u), v\right) & \text { for }(t, u, v) \in J \times \mathbb{R} \times\left(\left(-\infty,-\frac{1}{n}\right) \cup\left(\frac{1}{n}, \infty\right)\right), \\ \frac{1}{2} n\left[f\left(t, \chi_{n}(u), \frac{1}{n}\right)\left(v+\frac{1}{n}\right)-f\left(t, \chi_{n}(u),-\frac{1}{n}\right)\left(v-\frac{1}{n}\right)\right] \\ \text { for }(t, u, v) \in J \times \mathbb{R} \times\left[-\frac{1}{n}, \frac{1}{n}\right] .\end{cases}
$$


Then the inequalities

$$
a \leqslant-f_{n}(t, u, v)
$$

and

$$
-f_{n}(t, u, v) \leqslant \varphi(t)\left[h_{1}(u)+h_{1}(1)+h_{2}(u)\right]\left[\omega_{1}(g(|v|)+g(1))+\omega_{2}(g(|v|))\right]
$$

are satisfied for a.e. $t \in J$ and each $(u, v) \in \mathbb{R}_{+} \times \mathbb{R}_{0}$.

Consider the family of regular differential equations

$$
\left(g\left(x^{\prime}(t)\right)\right)^{\prime}=\mu f_{n}\left(t, x(t), x^{\prime}(t)\right)
$$

depending on the parameters $\mu \in \mathbb{R}$ and $n \in \mathbb{N}$.

Lemma 2.1. Let $A \in \mathbb{R}_{+}$and let $x$ be a solution of $B V P$ (2.3), (1.2), (1.3) with some $\mu=\mu_{x}$ and $n \in \mathbb{N}$ in (2.3). Then $\mu_{x}>0, x^{\prime}$ is decreasing on $J$,

$$
\left.\begin{array}{ll}
x^{\prime}(t) \geqslant g^{-1}\left(a \mu_{x}(\xi-t)\right) & \text { for } t \in[0, \xi], \\
x^{\prime}(t) \leqslant-g^{-1}\left(a \mu_{x}(t-\xi)\right) & \text { for } t \in(\xi, T],
\end{array}\right\}
$$

where $g^{-1}: \mathbb{R} \rightarrow \mathbb{R}$ denotes the inverse function to $g$ and $\xi \in(0, T)$ is the (unique) zero of $x^{\prime}$,

$$
x(t) \geqslant \begin{cases}\frac{A}{\xi} t & \text { for } t \in[0, \xi], \\ \frac{A}{T-\xi}(T-t) & \text { for } t \in(\xi, T],\end{cases}
$$

and

$$
\mu_{x} \leqslant \frac{1}{a}\left(A\left(1+\frac{1}{\beta}\right)\right)^{\beta}\left(\frac{2}{T}\right)^{1+\beta} .
$$

Proof. If $\mu_{x} \leqslant 0$, then $\left(g\left(x^{\prime}\right)\right)^{\prime} \geqslant-a \mu_{x} \geqslant 0$ a.e. on $J$ and so $g\left(x^{\prime}\right)$ is non-decreasing on $J$, which implies that $x^{\prime}$ is non-decreasing on $J$ as well. Since, by (1.3), $x^{\prime}\left(t_{0}\right)=0$ for a $t_{0} \in(0, T)$, we have $x^{\prime} \leqslant 0$ on $\left[0, t_{0}\right]$ and $x^{\prime} \geqslant 0$ on $\left[t_{0}, T\right]$ and then (1.2) yields $x \leqslant 0$ on $J$, contrary to (1.3). Hence $\mu_{x}>0$. Consequently, $\left(g\left(x^{\prime}\right)\right)^{\prime} \leqslant-a \mu_{x}<0$ a.e. on $J$, from which we deduce that $x^{\prime}$ is decreasing on $J$ and $x^{\prime}$ has the unique zero $\xi \in(0, T)$.

Using $g(0)=0, x^{\prime}(\xi)=0$ and integrating $\left(g\left(x^{\prime}\right)\right)^{\prime} \leqslant-a \mu_{x}$ over $[t, \xi] \subset[0, \xi]$ and $[\xi, t] \subset[\xi, T]$, we obtain $(2.4)$.

Since $x(0)=x(T)=0, x(\xi)=A$ and $x$ is concave on $J$, which follows from the fact that $x^{\prime}$ is decreasing on $J, x$ satisfies $(2.5)$.

It remains to prove $(2.6)$. Since $g(u) \leqslant u^{\beta}$ for $u \in \mathbb{R}_{+}$by $\left(\mathrm{H}_{1}\right)$, we have

$$
g^{-1}(u) \geqslant \sqrt[\beta]{u} \text { for } u \in \mathbb{R}_{+} .
$$

Therefore (see (2.4)),

$$
\begin{aligned}
A & =x(\xi)=\int_{0}^{\xi} x^{\prime}(t) \mathrm{d} t \geqslant \int_{0}^{\xi} g^{-1}\left(a \mu_{x}(\xi-t)\right) \mathrm{d} t=\frac{1}{a \mu_{x}} \int_{0}^{a \mu_{x} \xi} g^{-1}(t) \mathrm{d} t \\
& \geqslant \frac{1}{a \mu_{x}} \int_{0}^{a \mu_{x} \xi} \sqrt[\beta]{t} \mathrm{~d} t=\frac{\sqrt[\beta]{a \mu_{x}} \xi^{1+(1 / \beta)}}{1+(1 / \beta)}
\end{aligned}
$$


and

$$
\begin{aligned}
A & =x(\xi)=\int_{T}^{\xi} x^{\prime}(t) \mathrm{d} t \geqslant \int_{\xi}^{T} g^{-1}\left(a \mu_{x}(t-\xi)\right) \mathrm{d} t=\frac{1}{a \mu_{x}} \int_{0}^{a \mu_{x}(T-\xi)} g^{-1}(t) \mathrm{d} t \\
& \geqslant \frac{1}{a \mu_{x}} \int_{0}^{a \mu_{x}(T-\xi)} \sqrt[\beta]{t} \mathrm{~d} t=\frac{\sqrt[\beta]{a \mu_{x}}(T-\xi)^{1+(1 / \beta)}}{1+(1 / \beta)}
\end{aligned}
$$

Hence

$$
A \geqslant \frac{\sqrt[\beta]{a \mu_{x}}}{1+(1 / \beta)} \max \left\{\xi^{1+(1 / \beta)},(T-\xi)^{1+(1 / \beta)}\right\} \geqslant \frac{\sqrt[\beta]{a \mu_{x}}}{1+(1 / \beta)}\left(\frac{1}{2} T\right)^{1+(1 / \beta)}
$$

and then from the inequality

$$
\sqrt[\beta]{a \mu_{x}} \leqslant A\left(1+\frac{1}{\beta}\right)\left(\frac{2}{T}\right)^{1+(1 / \beta)}
$$

we deduce (2.6).

Lemma 2.2. Let $A \in \mathbb{R}_{+}$. Then there exists a positive constant $P$ independent of $n \in \mathbb{N}$ and $\lambda \in(0,1]$ such that for any solution $x$ of $B V P$ (2.3), (1.2) with some $\mu=\mu_{x}$ and $n \in \mathbb{N}$ in (2.3) satisfying

$$
\max \{x(t): t \in J\}=\lambda A, \quad \lambda \in(0,1],
$$

the inequalities

$$
\left\|x^{\prime}\right\|<P
$$

and

$$
0<\mu_{x} \leqslant \frac{1}{a}\left(A\left(1+\frac{1}{\beta}\right)\right)^{\beta}\left(\frac{2}{T}\right)^{1+\beta}
$$

are valid.

Proof. Let $x$ be a solution of BVP (2.3), (1.2) with some $\mu=\mu_{x}$ and $n \in \mathbb{N}$ in (2.3) satisfying (2.8). Then, by Lemma 2.1 (with $\lambda A$ instead of $A$ ),

$$
0<\mu_{x} \leqslant \frac{1}{a}\left(\lambda A\left(1+\frac{1}{\beta}\right)\right)^{\beta}\left(\frac{2}{T}\right)^{1+\beta} \leqslant \frac{1}{a}\left(A\left(1+\frac{1}{\beta}\right)\right)^{\beta}\left(\frac{2}{T}\right)^{1+\beta},
$$

$x>0$ on $(0, T), x^{\prime}$ is decreasing on $J$ and has the unique zero $\xi \in(0, T)$. Hence

$$
\left\|x^{\prime}\right\|=\max \left\{x^{\prime}(0),-x^{\prime}(T)\right\}
$$

and

$$
x(\xi)=\lambda A .
$$


In addition, by (2.2),

$$
\left(g\left(x^{\prime}(t)\right)\right)^{\prime} \geqslant-\mu_{x} \varphi(t)\left[h_{1}(x(t))+h_{1}(1)+h_{2}(x(t))\right]\left[\omega_{1}\left(g\left(\left|x^{\prime}(t)\right|\right)+g(1)\right)+\omega_{2}\left(g\left(\left|x^{\prime}(t)\right|\right)\right)\right]
$$

for a.e. $t \in J$. Consequently,

$$
\frac{\left(g\left(x^{\prime}(t)\right)\right)^{\prime} x^{\prime}(t)}{\omega_{1}\left(g\left(x^{\prime}(t)\right)+g(1)\right)+\omega_{2}\left(g\left(x^{\prime}(t)\right)\right)} \geqslant-\mu_{x} \varphi(t)\left[h_{1}(x(t))+h_{1}(1)+h_{2}(x(t))\right] x^{\prime}(t)
$$

for a.e. $t \in[0, \xi]$, and

$$
\frac{\left(g\left(x^{\prime}(t)\right)\right)^{\prime} x^{\prime}(t)}{\omega_{1}\left(-g\left(x^{\prime}(t)\right)+g(1)\right)+\omega_{2}\left(-g\left(x^{\prime}(t)\right)\right)} \leqslant-\mu_{x} \varphi(t)\left[h_{1}(x(t))+h_{1}(1)+h_{2}(x(t))\right] x^{\prime}(t)
$$

for a.e. $t \in[\xi, T]$. Integrating $(2.14)$ over $[0, \xi]$ and $(2.15)$ over $[\xi, T]$, we get

$$
\begin{aligned}
\int_{0}^{g\left(x^{\prime}(0)\right)} \frac{g^{-1}(s)}{\omega_{1}(s+g(1))}+\omega_{2}(s) & \mathrm{d} s \\
& =-\int_{0}^{\xi} \frac{\left(g\left(x^{\prime}(t)\right)\right)^{\prime} x^{\prime}(t)}{\omega_{1}\left(g\left(x^{\prime}(t)\right)+g(1)\right)+\omega_{2}\left(g\left(x^{\prime}(t)\right)\right)} \mathrm{d} t \\
& \leqslant \mu_{x} \int_{0}^{\xi} \varphi(t)\left[h_{1}(x(t))+h_{1}(1)+h_{2}(x(t))\right] x^{\prime}(t) \mathrm{d} t \\
& \leqslant \mu_{x}\|\varphi\|_{\infty}\left(h_{1}(1) x(\xi)+\int_{0}^{x(\xi)}\left(h_{1}(t)+h_{2}(t)\right) \mathrm{d} t\right) \\
& \leqslant \mu_{x}\|\varphi\|_{\infty}\left(h_{1}(1) A+\int_{0}^{A}\left(h_{1}(t)+h_{2}(t)\right) \mathrm{d} t\right)
\end{aligned}
$$

and

$$
\begin{aligned}
\int_{0}^{g\left(-x^{\prime}(T)\right)} \frac{g^{-1}(s)}{\omega_{1}(s+g(1))}+\omega_{2}(s) & \mathrm{d} s \\
= & \int_{\xi}^{T} \frac{\left(g\left(x^{\prime}(t)\right)\right)^{\prime} x^{\prime}(t)}{\omega_{1}\left(-g\left(x^{\prime}(t)\right)+g(1)\right)+\omega_{2}\left(-g\left(x^{\prime}(t)\right)\right)} \mathrm{d} t \\
& \leqslant-\mu_{x} \int_{\xi}^{T} \varphi(t)\left[h_{1}(x(t))+h_{1}(1)+h_{2}(x(t))\right] x^{\prime}(t) \mathrm{d} t \\
& \leqslant-\mu_{x}\|\varphi\|_{\infty}\left(-h_{1}(1) x(\xi)-\int_{0}^{x(\xi)}\left(h_{1}(t)+h_{2}(t)\right) \mathrm{d} t\right) \\
& \leqslant \mu_{x}\|\varphi\|_{\infty}\left(h_{1}(1) A+\int_{0}^{A}\left(h_{1}(t)+h_{2}(t)\right) \mathrm{d} t\right) .
\end{aligned}
$$

Since

$$
\int_{0}^{\infty} \frac{\sqrt[\beta]{u}}{\omega_{1}(u)} \mathrm{d} u=\infty
$$


by $\left(\mathrm{H}_{3}\right)$, from the properties of $\omega_{1}$ and using (2.7) we have

$$
\int_{0}^{\infty} \frac{g^{-1}(u)}{\omega_{1}(u+g(1))} \mathrm{d} u=\infty
$$

and then from the fact that $\omega_{2}: \mathbb{R}_{+} \rightarrow \mathbb{R}_{+}$is non-increasing we deduce that

$$
\int_{0}^{\infty} \frac{g^{-1}(u)}{\omega_{1}(u+g(1))+\omega_{2}(u)} \mathrm{d} u=\infty .
$$

Hence there exists a positive constant $Q$ such that

$$
\int_{0}^{Q} \frac{g^{-1}(u)}{\omega_{1}(u+g(1))+\omega_{2}(u)} \mathrm{d} u>\mu_{x}\|\varphi\|_{\infty}\left(h_{1}(1) A+\int_{0}^{A}\left(h_{1}(u)+h_{2}(u)\right) \mathrm{d} u\right) .
$$

Then (2.16) and (2.17) give

$$
\max \left\{g\left(x^{\prime}(0)\right), g\left(-x^{\prime}(T)\right)\right\}<Q,
$$

and from (2.11) we see that (2.9) is satisfied with $P=g^{-1}(Q)$.

Lemma 2.3. Let $A \in \mathbb{R}_{+}$. Then there exists a positive constant $\mu_{A}$ independent of $n \in \mathbb{N}$ such that for any solution $x$ of $B V P$ (2.3), (1.2), (1.3) with some $\mu=\mu_{x}$ and $n \in \mathbb{N}$ in (2.3), the inequality

$$
\mu_{A} \leqslant \mu_{x}
$$

is satisfied.

Proof. Let $x$ be a solution of BVP (2.3), (1.2), (1.3) with some $\mu=\mu_{x}$ and $n \in \mathbb{N}$ in (2.3). Then $x(\xi)=A$, where $\xi \in(0, T)$ is the unique zero of $x^{\prime}$ (see Lemma 2.1). Whence

$$
A=x(\xi)-x(0)=x^{\prime}\left(\eta_{1}\right) \xi, \quad A=x(\xi)-x(T)=-x^{\prime}\left(\eta_{2}\right)(T-\xi),
$$

where $0<\eta_{1}<\xi<\eta_{2}<T$, and so $x^{\prime}\left(\eta_{1}\right)=A / \xi,-x^{\prime}\left(\eta_{2}\right)=A /(T-\xi)$. Since $\min \{\xi, T-\xi\} \leqslant T / 2$, we have $\max \left\{x^{\prime}\left(\eta_{1}\right),-x^{\prime}\left(\eta_{2}\right)\right\} \geqslant 2 A / T$ and then $\left\|x^{\prime}\right\| \geqslant 2 A / T$. Now arguing as in the proof of Lemma 2.2 (see (2.16) and (2.17)),

$$
\begin{aligned}
\int_{0}^{g(2 A / T)} \frac{g^{-1}(s)}{\omega_{1}(s+g(1))+\omega_{2}(s)} \mathrm{d} s & \leqslant \int_{0}^{g\left(\left\|x^{\prime}\right\|\right)} \frac{g^{-1}(s)}{\omega_{1}(s+g(1))+\omega_{2}(s)} \mathrm{d} s \\
& \leqslant \mu_{x}\|\varphi\|_{\infty}\left(h_{1}(1) A+\int_{0}^{A}\left(h_{1}(s)+h_{2}(s)\right) \mathrm{d} s\right) .
\end{aligned}
$$

Thus (2.18) holds with

$$
\mu_{A}=\left(\int_{0}^{g(2 A / T)} \frac{g^{-1}(s)}{\omega_{1}(s+g(1))+\omega_{2}(s)} \mathrm{d} s\right) /\left(\|\varphi\|_{\infty}\left(h_{1}(1) A+\int_{0}^{A}\left(h_{1}(s)+h_{2}(s)\right) \mathrm{d} s\right)\right) .
$$


Lemma 2.4. Let $A \in \mathbb{R}_{+}$. Then $B V P(2.3)$, (1.2), (1.3) has a solution for each $n \in \mathbb{N}$.

Proof. Fix $n \in \mathbb{N}$ and let $P \in \mathbb{R}_{+}$be given by Lemma 2.2. Set

$$
\begin{aligned}
& \Omega=\left\{(x, \mu):(x, \mu) \in C^{1}(J ; \mathbb{R}) \times \mathbb{R},\|x\|\right.<A+1,\left\|x^{\prime}\right\|<P \\
&\left.|\mu|<\frac{1}{a}\left(A\left(1+\frac{1}{\beta}\right)\right)^{\beta}\left(\frac{2}{T}\right)^{1+\beta}+1\right\} .
\end{aligned}
$$

Then $\Omega$ is an open, bounded and symmetric with respect to $(0,0)$ subset of the Banach space $C^{1}(J ; \mathbb{R}) \times \mathbb{R}$. Define $\mathcal{H}:[0,1] \times \bar{\Omega} \rightarrow C^{1}(J ; \mathbb{R}) \times \mathbb{R}$ by

$$
\begin{aligned}
& \mathcal{H}(\lambda, x, \mu)=\left\{\int_{0}^{t} g^{-1}\left(B+\mu\left((\lambda-1) s+\lambda \int_{0}^{s} f_{n}\left(\tau, x(\tau), x^{\prime}(\tau)\right) \mathrm{d} \tau\right)\right) \mathrm{d} s,\right. \\
&\left.\lambda[\max \{x(t): t \in J\}+\min \{x(t): t \in J\}]+(1-\lambda) x\left(\frac{1}{2} T\right)+\mu\right\},
\end{aligned}
$$

where the constant $B=B(\lambda, x, \mu)$ is the unique solution of the equation

$$
p(c ; \lambda, x, \mu)=0
$$

with

$$
p(c ; \lambda, x, \mu)=\int_{0}^{T} g^{-1}\left(c+\mu\left((\lambda-1) t+\lambda \int_{0}^{t} f_{n}\left(s, x(s), x^{\prime}(s)\right) \mathrm{d} s\right)\right) \mathrm{d} t .
$$

The existence and uniqueness of a solution for (2.19) follows from the fact that $p(\cdot ; \lambda, x, \mu)$ is continuous and increasing on $\mathbb{R}$ and

$$
\lim _{c \rightarrow-\infty} p(c ; \lambda, x, \mu)=-\infty, \quad \lim _{c \rightarrow \infty} p(c ; \lambda, x, \mu)=\infty
$$

for each $(\lambda, x, \mu) \in[0,1] \times \bar{\Omega}$.

Since

$$
\mathcal{H}(0, x, \mu)=\left\{\int_{0}^{t} g^{-1}(B-\mu s) \mathrm{d} s, x\left(\frac{1}{2} T\right)+\mu\right\},
$$

where $B$ is the unique solution of the equation $p(c ; 0, x, \mu)=0$, that is the equation

$$
\int_{0}^{T} g^{-1}(c-\mu t) \mathrm{d} t=0
$$

the mean-value theorem for integrals gives $B=\mu t_{0}$ for a $t_{0} \in(0, T)$. Hence

$$
\mathcal{H}(0, x, \mu)=\left\{\int_{0}^{t} g^{-1}\left(\mu\left(t_{0}-s\right)\right) \mathrm{d} s, x\left(\frac{1}{2} T\right)+\mu\right\},
$$

and we see that $\mathcal{H}(0,-x,-\mu)=-\mathcal{H}(0, x, \mu)$ for $(x, \mu) \in \bar{\Omega}$. Consequently, $\mathcal{H}(0, \cdot, \cdot)$ is an odd operator. 
We are going to show that $\mathcal{H}$ is a compact operator. Let

$$
\left\{\left(\lambda_{m}, x_{m}, \mu_{m}\right)\right\} \subset[0,1] \times \bar{\Omega} \text { and } \lim _{m \rightarrow \infty}\left(\lambda_{m}, x_{m}, \mu_{m}\right)=\left(\lambda_{0}, x_{0}, \mu_{0}\right)
$$

in $[0,1] \times C^{1}(J ; \mathbb{R}) \times \mathbb{R}$. Let $B_{m}$ be the (unique) solution of the equation $p\left(c ; \lambda_{m}, x_{m}, \mu_{m}\right)=$ 0 , where the function $p$ is given by $(2.20)$. Since $\left|f_{n}\left(t, x_{m}(t), x_{m}^{\prime}(t)\right)\right| \leqslant q(t)$ for a.e. $t \in J$ and each $m \in \mathbb{N}$ with a $q \in L_{1}(J ; \mathbb{R})$, we see that $\left\{B_{m}\right\}$ is bounded. If $\left\{B_{m}\right\}$ is not convergent, there exist convergent subsequences $\left\{B_{k_{m}}\right\}$ and $\left\{B_{l_{m}}\right\}$ such that $\lim _{m \rightarrow \infty} B_{k_{m}}=c_{1}$, $\lim _{m \rightarrow \infty} B_{l_{m}}=c_{2}$ and $c_{1} \neq c_{2}$. Then applying the Lebesgue dominated convergence theorem, we have

$$
0=\lim _{m \rightarrow \infty} p\left(B_{k_{m}} ; \lambda_{k_{m}}, x_{k_{m}}, \mu_{k_{m}}\right)=p\left(c_{1} ; \lambda_{0}, x_{0}, \mu_{0}\right)
$$

and

$$
0=\lim _{m \rightarrow \infty} p\left(B_{l_{m}} ; \lambda_{l_{m}}, x_{l_{m}}, \mu_{l_{m}}\right)=p\left(c_{2} ; \lambda_{0}, x_{0}, \mu_{0}\right),
$$

which contradicts $p\left(c_{1} ; \lambda_{0}, x_{0}, \mu_{0}\right) \neq p\left(c_{2} ; \lambda_{0}, x_{0}, \mu_{0}\right)$. Hence $\left\{B_{m}\right\}$ is convergent and let $\lim _{m \rightarrow \infty} B_{m}=B_{0}$. Then

$$
\begin{aligned}
\lim _{m \rightarrow \infty} \int_{0}^{t} g^{-1}\left(B_{m}\right. & \left.+\mu_{m}\left(\left(\lambda_{m}-1\right) s+\lambda_{m} \int_{0}^{s} f_{n}\left(\tau, x_{m}(\tau), x_{m}^{\prime}(\tau)\right) \mathrm{d} \tau\right)\right) \mathrm{d} s \\
= & \int_{0}^{t} g^{-1}\left(B_{0}+\mu_{0}\left(\left(\lambda_{0}-1\right) s+\lambda_{0} \int_{0}^{s} f_{n}\left(\tau, x_{0}(\tau), x_{0}^{\prime}(\tau)\right) \mathrm{d} \tau\right)\right) \mathrm{d} s
\end{aligned}
$$

in $C^{1}(J)$, and since

$$
\begin{aligned}
\lim _{m \rightarrow \infty}\left(\lambda_{m}\left[\max \left\{x_{m}(t): t \in J\right\}+\min \left\{x_{m}(t): t \in J\right\}\right]+\left(1-\lambda_{m}\right) x_{m}\left(\frac{1}{2} T\right)+\mu_{m}\right) \\
=\lambda_{0}\left[\max \left\{x_{0}(t): t \in J\right\}+\min \left\{x_{0}(t): t \in J\right\}\right]+\left(1-\lambda_{0}\right) x_{0}\left(\frac{1}{2} T\right)+\mu_{0},
\end{aligned}
$$

$\mathcal{H}$ is a continuous operator. Let $\left\{\left(\lambda_{j}, x_{j}, \mu_{j}\right)\right\} \subset[0,1 \times \bar{\Omega}]$. Then the sequence

$$
\left\{\lambda_{j}\left[\max \left\{x_{j}(t): t \in J\right\}+\min \left\{x_{j}(t): t \in J\right\}\right]+\left(1-\lambda_{j}\right) x_{j}\left(\frac{1}{2} T\right)+\mu_{j}\right\}
$$

is bounded and there exists $r \in L_{1}(J ; \mathbb{R})$ such that $\left|f_{n}\left(t, x_{j}(t), x_{j}^{\prime}(t)\right)\right| \leqslant r(t)$ for a.e. $t \in J$ and each $j \in \mathbb{N}$, and so $\left\{B_{j}\right\}$ is bounded where $B_{j}$ is the unique solution of the equation $p\left(c ; \lambda_{j}, x_{j}, \mu_{j}\right)=0$. Hence

$$
\left\{\int_{0}^{t} g^{-1}\left(B_{j}+\mu_{j}\left(\left(\lambda_{j}-1\right) s+\lambda_{j} \int_{0}^{s} f_{n}\left(\tau, x_{j}(\tau), x_{j}^{\prime}(\tau)\right) \mathrm{d} \tau\right)\right) \mathrm{d} s\right\}
$$

is bounded in $C^{1}(J ; \mathbb{R})$ and

$$
\left\{B_{j}+\mu_{j}\left(\left(\lambda_{j}-1\right) t+\lambda_{j} \int_{0}^{t} f_{n}\left(s, x_{j}(s), x_{j}^{\prime}(s)\right) \mathrm{d} s\right)\right\}
$$


is equicontinuous on $J$. So $\left\{\mathcal{H}\left(\lambda_{j}, x_{j}, \mu_{j}\right)\right\}$ is relatively compact in $C^{1}(J ; \mathbb{R}) \times \mathbb{R}$ by the Arzelà-Ascoli theorem and the Bolzano-Weierstrass theorem. Finally, let

$$
\mathcal{H}\left(\lambda_{0}, x_{0}, \mu_{0}\right)=\left(x_{0}, \mu_{0}\right)
$$

for some $\left(\lambda_{0}, x_{0}, \mu_{0}\right) \in[0,1] \times \partial \Omega$. Then from the definition of the operator $\mathcal{H}$ we deduce that

$$
\begin{gathered}
\left(g\left(x_{0}^{\prime}(t)\right)\right)^{\prime}=\mu_{0}\left[\lambda_{0}-1+\lambda_{0} f_{n}\left(t, x_{0}(t), x_{0}^{\prime}(t)\right)\right] \quad \text { for a.e. } t \in J \\
x_{0}(0)=0, \quad x_{0}(T)=0
\end{gathered}
$$

and

$$
\lambda_{0}\left[\max \left\{x_{0}(t): t \in J\right\}+\min \left\{x_{0}(t): t \in J\right\}\right]+\left(1-\lambda_{0}\right) x_{0}\left(\frac{1}{2} T\right)=0 .
$$

If $\mu_{0}>0$, then $(2.21)$ gives $\left(g\left(x_{0}^{\prime}\right)\right)^{\prime}<0$ a.e. on $J$, and using $(2.22)$ we get $x_{0}>0$ on $(0, T)$, contrary to (see $(2.23))$

$$
\begin{aligned}
0 & =\lambda_{0}\left[\max \left\{x_{0}(t): t \in J\right\}+\min \left\{x_{0}(t): t \in J\right\}\right]+\left(1-\lambda_{0}\right) x_{0}\left(\frac{1}{2} T\right) \\
& =\lambda_{0} \max \left\{x_{0}(t): t \in J\right\}+\left(1-\lambda_{0}\right) x_{0}\left(\frac{1}{2} T\right)>0 .
\end{aligned}
$$

Let $\mu_{0}<0$. Then from $(2.21)$ it follows that $\left(g\left(x_{0}^{\prime}\right)\right)^{\prime}>0$ a.e. on $J$ which, together with $(2.22)$, yields $x_{0}<0$ on $(0, T)$, contrary to (see $(2.23)$ )

$$
\begin{aligned}
0 & =\lambda_{0}\left[\max \left\{x_{0}(t): t \in J\right\}+\min \left\{x_{0}(t): t \in J\right\}\right]+\left(1-\lambda_{0}\right) x_{0}\left(\frac{1}{2} T\right) \\
& =\lambda_{0} \min \left\{x_{0}(t): t \in J\right\}+\left(1-\lambda_{0}\right) x_{0}\left(\frac{1}{2} T\right)<0 .
\end{aligned}
$$

Hence $\mu_{0}=0$ and then from $\left(g\left(x_{0}^{\prime}\right)\right)^{\prime}=0$ a.e. on $J$ and $(2.22)$ we see that $x_{0}=0$, contrary to $(0,0)=\left(x_{0}, \mu_{0}\right) \in \partial \Omega$. We have proved that $\mathcal{H}(\lambda, x, \mu) \neq(x, \mu)$ for $\lambda \in[0,1]$ and $(x, \mu) \in \partial \Omega$. Therefore, by the Borsuk antipodal theorem, $D(\mathcal{I}-\mathcal{H}(0, \cdot, \cdot), \Omega, 0) \neq$ 0 , where ' $D$ ' stands for the Leray-Schauder degree and $\mathcal{I}$ is the identity operator on $C^{1}(J ; \mathbb{R}) \times \mathbb{R}$. In addition,

$$
D(\mathcal{I}-\mathcal{H}(1, \cdot, \cdot), \Omega, 0)=D(\mathcal{I}-\mathcal{H}(0, \cdot, \cdot), \Omega, 0)
$$

by the homotopy invariance property. Consequently,

$$
D(\mathcal{I}-\mathcal{H}(1, \cdot, \cdot), \Omega, 0) \neq 0 .
$$

Finally, define the operator $\mathcal{K}:[0,1] \times \bar{\Omega} \rightarrow C^{1}(J ; \mathbb{R}) \times \mathbb{R}$ by

$$
\begin{aligned}
\mathcal{K}(\lambda, x, \mu)=\left\{\int _ { 0 } ^ { t } g ^ { - 1 } \left(C+\mu \int_{0}^{s}\right.\right. & \left.f_{n}\left(\tau, x(\tau), x^{\prime}(\tau)\right) \mathrm{d} \tau\right) \mathrm{d} s, \\
& \max \{x(t): t \in J\}+\min \{x(t): t \in J\}-\lambda A+\mu\},
\end{aligned}
$$


where the constant $C=C(\lambda, x, \mu)$ is the unique solution of the equation

$$
r(c ; \lambda, x, \mu)=0
$$

with

$$
r(c ; \lambda, x, \mu)=\int_{0}^{T} g^{-1}\left(c+\mu \int_{0}^{t} f_{n}\left(s, x(s), x^{\prime}(s)\right) \mathrm{d} s\right) \mathrm{d} t .
$$

The existence and uniqueness of a solution of (2.25) can be verified in the same way as that for (2.19). Arguing as in the case of the operator $\mathcal{H}$, it may be proved that $\mathcal{K}$ is a compact operator. Assume that $\mathcal{K}\left(\lambda_{*}, x_{*}, \mu_{*}\right)=\left(x_{*}, \mu_{*}\right)$ for a $\left(\lambda_{*}, x_{*}, \mu_{*}\right) \in[0,1] \times \partial \Omega$. Then

$$
\begin{gathered}
\left(g\left(x_{*}^{\prime}(t)\right)\right)^{\prime}=\mu_{*} f_{n}\left(t, x_{*}(t), x_{*}^{\prime}(t)\right) \quad \text { for a.e. } t \in J, \\
x_{*}(0)=0, \quad x_{*}(T)=0
\end{gathered}
$$

and

$$
\max \left\{x_{*}(t): t \in J\right\}+\min \left\{x_{*}(t): t \in J\right\}=\lambda_{*} A .
$$

If $\mu_{*} \leqslant 0$, then $\left(g\left(x_{*}^{\prime}\right)\right)^{\prime} \geqslant 0$ a.e. on $J$ by $(2.27)$, and using $(2.28)$ we deduce that $x_{*} \leqslant 0$ on $J$. Hence $\max \left\{x_{*}(t): t \in J\right\}=0$ and (see (2.29))

$$
0 \leqslant \lambda_{*} A=\max \left\{x_{*}(t): t \in J\right\}+\min \left\{x_{*}(t): t \in J\right\}=\min \left\{x_{*}(t): t \in J\right\}
$$

leads to $x_{*}=0$. Then $\mu_{*}=0$, which follows from $(2.1)$ and $(2.27)$, contrary to $(0,0)=$ $\left(x_{*}, \mu_{*}\right) \in \partial \Omega$. Whence $\mu_{*}>0$, and then, from $\left(g\left(x_{*}^{\prime}\right)\right)^{\prime}<0$ a.e. on $J$ and $(2.28)$, we conclude that $x_{*}>0$ on $(0, T)$ and $(2.29)$ yields $\max \left\{x_{*}(t): t \in J\right\}=\lambda_{*} A$. So $x_{*}$ is a solution of BVP (2.3), (1.2), (2.8) (with $\mu=\mu_{*}$ in (2.3) and $\lambda=\lambda_{*}$ in (2.8)). Consequently,

$$
\left\|x_{*}^{\prime}\right\|<P, \quad\left|\mu_{*}\right| \leqslant \frac{1}{a}\left(A\left(1+\frac{1}{\beta}\right)\right)^{\beta}\left(\frac{2}{T}\right)^{1+\beta}
$$

by Lemma 2.2 , and $\left\|x_{*}\right\|=\lambda_{*} A \leqslant A$. Hence $\left(x_{*}, \mu_{*}\right) \notin \partial \Omega$ and we have proved that $\mathcal{K}(\lambda, x, \mu) \neq(x, \mu)$ for $\lambda \in[0,1]$ and $(x, \mu) \in \partial \Omega$. Now, by the homotopy invariance property,

$$
D(\mathcal{I}-\mathcal{K}(0, \cdot, \cdot) \Omega, 0)=D(\mathcal{I}-\mathcal{K}(1, \cdot, \cdot) \Omega, 0),
$$

and since $\mathcal{H}(1, \cdot, \cdot)=\mathcal{K}(0, \cdot, \cdot),(2.24)$ gives

$$
D(\mathcal{I}-\mathcal{K}(1, \cdot, \cdot), \Omega, 0) \neq 0 .
$$

Then there exists a fixed point $(\hat{x}, \hat{\mu})$ of the operator $\mathcal{K}(1, \cdot, \cdot)$ and it is easy to check that $\hat{x}$ is a solution of $\operatorname{BVP}(2.3),(1.2),(1.3)$ with $\mu=\hat{\mu}$ in $(2.3)$.

Lemma 2.5. Let $A \in \mathbb{R}_{+}$and let $x_{n}$ be a solution of $B V P(2.3),(1.2),(1.3)$ with $n \in \mathbb{N}$ in (2.3) and let $\xi_{n} \in(0, T)$ be the (unique) zero of $x_{n}^{\prime}$. Then there exist $0<c_{1}<c_{2}<T$ such that

$$
c_{1}<\xi_{n}<c_{2} \quad \text { for } n \in \mathbb{N} \text {. }
$$


Proof. By Lemma 2.2, there exists a positive constant $P$ such that $\left\|x_{n}^{\prime}\right\|<P$ for $n \in \mathbb{N}$. Since $x_{n}\left(\xi_{n}\right)=A$, we have

$$
A=x_{n}\left(\xi_{n}\right)-x_{n}(0)=x_{n}^{\prime}\left(\tau_{n}\right) \xi_{n}, \quad A=x_{n}\left(\xi_{n}\right)-x_{n}(T)=x_{n}^{\prime}\left(\varrho_{n}\right)\left(\xi_{n}-T\right),
$$

where $0<\tau_{n}<\xi_{n}<\varrho_{n}<T$. Then

$$
P>x_{n}^{\prime}\left(\tau_{n}\right)=\frac{A}{\xi_{n}}, \quad P>-x_{n}^{\prime}\left(\varrho_{n}\right)=\frac{A}{T-\xi_{n}},
$$

and so

$$
\frac{A}{P}<\xi_{n}<T-\frac{A}{P} \text { for } n \in \mathbb{N} .
$$

Hence (2.30) holds with $c_{1}=A / P$ and $c_{2}=T-A / P$.

Lemma 2.6. Let $A \in \mathbb{R}_{+}$. For each $n \in \mathbb{N}$, let $x_{n}$ be a solution of $B V P(2.3)$, (1.2), (1.3) with $n \in \mathbb{N}$ and $\mu=\mu_{n}$ in (2.3). Then the sequence

$$
\left\{f_{n}\left(t, x_{n}(t), x_{n}^{\prime}(t)\right)\right\} \subset L_{1}(J)
$$

is uniformly absolutely continuous (UAC) on $J$, that is, for each $\varepsilon>0$ there exists $\delta>0$ such that

$$
\int_{\mathcal{M}}\left|f_{n}\left(t, x_{n}(t), x_{n}^{\prime}(t)\right)\right| \mathrm{d} t<\varepsilon
$$

for $n \in \mathbb{N}$ whenever $\mathcal{M} \subset J$ is a measurable set and $\mu(\mathcal{M})<\delta$.

Proof. By Lemma $2.2($ for $n \in \mathbb{N}$ ),

$$
x_{n}(t) \geqslant \begin{cases}\frac{A}{\xi_{n}} t & \text { for } t \in\left[0, \xi_{n}\right], \\ \frac{A}{T-\xi_{n}}(T-t) & \text { for } t \in\left(\xi_{n}, T\right],\end{cases}
$$

where $\xi_{n} \in(0, T)$ is the unique zero of $x_{n}^{\prime}$ and

$$
\begin{aligned}
g\left(x_{n}^{\prime}(t)\right) \geqslant a \mu_{n}\left(\xi_{n}-t\right) & \text { for } t \in\left[0, \xi_{n}\right], \\
g\left(\left|x_{n}^{\prime}(t)\right|\right) \geqslant a \mu_{n}\left(t-\xi_{n}\right) & \text { for } t \in\left(\xi_{n}, T\right] .
\end{aligned}
$$

Next, by Lemmas 2.2, 2.3 and 2.5, there exist positive constants $P, \mu_{A}$ and $0<c_{1}<$ $c_{2}<T$ such that (for $n \in \mathbb{N}$ )

$$
\begin{gathered}
\left\|x_{n}^{\prime}\right\|<P, \\
\mu_{A} \leqslant \mu_{n} \leqslant \frac{1}{a}\left(A\left(1+\frac{1}{\beta}\right)\right)^{\beta}\left(\frac{2}{T}\right)^{1+\beta}
\end{gathered}
$$

and

$$
c_{1}<\xi_{n}<c_{2} .
$$


Set

$$
\Delta=\min \left\{\frac{A}{c_{2}}, \frac{A}{T-c_{1}}, a \mu_{A}\right\}
$$

Then $($ for $n \in \mathbb{N}$ )

$$
x_{n}(t) \geqslant \begin{cases}\Delta t & \text { for } t \in\left[0, \xi_{n}\right], \\ \Delta(T-t) & \text { for } t \in\left(\xi_{n}, T\right]\end{cases}
$$

and

$$
\left.\begin{array}{rl}
g\left(x_{n}^{\prime}(t)\right) \geqslant \Delta\left(\xi_{n}-t\right) & \text { for } t \in\left[0, \xi_{n}\right], \\
g\left(\left|x_{n}^{\prime}(t)\right|\right) \geqslant \Delta\left(t-\xi_{n}\right) & \text { for } t \in\left(\xi_{n}, T\right] .
\end{array}\right\}
$$

Next (2.2) gives

$$
\left|f_{n}\left(t, x_{n}(t), x_{n}^{\prime}(t)\right)\right| \leqslant\|\varphi\|_{\infty}\left[S_{1}+h_{2}\left(x_{n}(t)\right)\right]\left[S_{2}+\omega_{2}\left(g\left(\left|x_{n}^{\prime}(t)\right|\right)\right)\right]
$$

for a.e. $t \in J$ and each $n \in \mathbb{N}$, where

$$
S_{1}=h_{1}(A), \quad S_{2}=\omega_{1}(g(P)) .
$$

Hence to prove our lemma it suffices to verify that the sequences

$$
\left\{h_{2}\left(x_{n}(t)\right)\right\}, \quad\left\{\omega_{2}\left(g\left(\left|x_{n}^{\prime}(t)\right|\right)\right)\right\}, \quad\left\{h_{2}\left(x_{n}(t)\right) \omega_{2}\left(g\left(\left|x_{n}^{\prime}(t)\right|\right)\right)\right\}
$$

are UAC on $J$. From the structure of measurable sets in $\mathbb{R}$ we deduce that the sequences (2.35) are UAC on $J$ if for each $\varepsilon>0$ there exists $\delta>0$ such that for any at-mostcountable set $\left\{\left(a_{j}, b_{j}\right)\right\}_{j \in \mathbb{J}}$ of mutually disjoint intervals $\left(a_{j}, b_{j}\right) \subset J, \sum_{j \in \mathbb{J}}\left(b_{j}-a_{j}\right)<\delta$, we have

$$
\begin{gathered}
\sum_{j \in \mathbb{J}} \int_{a_{j}}^{b_{j}} h_{2}\left(x_{n}(t)\right) \mathrm{d} t<\varepsilon, \quad \sum_{j \in \mathbb{J}} \int_{a_{j}}^{b_{j}} \omega_{2}\left(g\left(\left|x_{n}^{\prime}(t)\right|\right)\right) \mathrm{d} t<\varepsilon, \\
\sum_{j \in \mathbb{J}} \int_{a_{j}}^{b_{j}} h_{2}\left(x_{n}(t)\right) \omega_{2}\left(g\left(\left|x_{n}^{\prime}(t)\right|\right)\right) \mathrm{d} t<\varepsilon
\end{gathered}
$$

for each $n \in \mathbb{N}$.

For this, let $\left\{\left(a_{j}, b_{j}\right)\right\}_{j \in \mathbb{J}}$ be an at-most-countable set of mutually disjoint intervals $\left(a_{j}, b_{j}\right) \subset J$. We first show that $\left\{h_{2}\left(x_{n}(t)\right)\right\}$ is UAC on $J$. Set

$$
\mathbb{J}_{n}^{1}=\left\{j: j \in \mathbb{J},\left(a_{j}, b_{j}\right) \subset\left(0, \xi_{n}\right)\right\}, \quad \mathbb{J}_{n}^{2}=\left\{j: j \in \mathbb{J},\left(a_{j}, b_{j}\right) \subset\left(\xi_{n}, T\right)\right\}
$$

for $n \in \mathbb{N}$. Then for $j \in \mathbb{J}_{n}^{1}$ and $i \in \mathbb{J}_{n}^{2}$ we have (see (2.33))

$$
\begin{aligned}
& \int_{a_{j}}^{b_{j}} h_{2}\left(x_{n}(t)\right) \mathrm{d} t \leqslant \int_{a_{j}}^{b_{j}} h_{2}(\Delta t) \mathrm{d} t=\frac{1}{\Delta} \int_{\Delta a_{j}}^{\Delta b_{j}} h_{2}(t) \mathrm{d} t \\
& \int_{a_{i}}^{b_{i}} h_{2}\left(x_{n}(t)\right) \mathrm{d} t \leqslant \int_{a_{i}}^{b_{i}} h_{2}(\Delta(T-t)) \mathrm{d} t=\frac{1}{\Delta} \int_{\Delta\left(T-b_{i}\right)}^{\Delta\left(T-a_{i}\right)} h_{2}(t) \mathrm{d} t .
\end{aligned}
$$


If $\mathbb{J} \neq \mathbb{J}_{n}^{1} \cup \mathbb{J}_{n}^{2}$ and $\left\{j_{0}\right\}=\mathbb{J} \backslash\left(\mathbb{J}_{n}^{1} \cup \mathbb{J}_{n}^{2}\right)$, that is $a_{j_{0}}<\xi_{n}<b_{j_{0}}$, then

$$
\begin{aligned}
\int_{a_{j_{0}}}^{b_{j_{0}}} h_{2}\left(x_{n}(t)\right) \mathrm{d} t & \leqslant \int_{a_{j_{0}}}^{\xi_{n}} h_{2}(\Delta t) \mathrm{d} t+\int_{\xi_{n}}^{b_{j_{0}}} h_{2}(\Delta(T-t)) \mathrm{d} t \\
& =\frac{1}{\Delta}\left[\int_{\Delta a_{j_{0}}}^{\Delta \xi_{n}} h_{2}(t) \mathrm{d} t+\int_{\Delta\left(T-b_{j_{0}}\right)}^{\Delta\left(T-\xi_{n}\right)} h_{2}(t) \mathrm{d} t\right] .
\end{aligned}
$$

Since $h_{2} \in L_{1}([0, \Delta T])$ and

$$
\sum_{j \in \mathbb{J}_{n}^{1}} \Delta\left(b_{j}-a_{j}\right)+\sum_{i \in \mathbb{J}_{n}^{2}} \Delta\left[\left(T-a_{i}\right)-\left(T-b_{i}\right)\right]+E_{n}=\Delta \sum_{j \in \mathbb{J}}\left(b_{j}-a_{j}\right),
$$

where

$$
E_{n}= \begin{cases}0 & \text { if } \mathbb{J}=\mathbb{J}_{n}^{1} \cup \mathbb{J}_{n}^{2} \\ \Delta\left(b_{j_{0}}-a_{j_{0}}\right) & \text { if }\left\{j_{0}\right\}=\mathbb{J} \backslash\left(\mathbb{J}_{n}^{1} \cup J_{n}^{2}\right),\end{cases}
$$

we conclude from (2.36)-(2.38) that $\left\{h_{2}\left(x_{n}(t)\right)\right\}$ is UAC on $J$.

We are going to show that $\left\{\omega_{2}\left(g\left(\left|x_{n}^{\prime}(t)\right|\right)\right)\right\}$ is UAC on $J$. For $j \in \mathbb{J}_{n}^{1}$ and $i \in \mathbb{J}_{n}^{2}$, it follows from (2.34) that

$$
\begin{aligned}
& \int_{a_{j}}^{b_{j}} \omega_{2}\left(g\left(\left|x_{n}^{\prime}(t)\right|\right)\right) \mathrm{d} t \leqslant \int_{a_{j}}^{b_{j}} \omega_{2}\left(\Delta\left(\xi_{n}-t\right)\right) \mathrm{d} t=\frac{1}{\Delta} \int_{\Delta\left(\xi_{n}-b_{j}\right)}^{\Delta\left(\xi_{n}-a_{j}\right)} \omega_{2}(t) \mathrm{d} t, \\
& \int_{a_{i}}^{b_{i}} \omega_{2}\left(g\left(\left|x_{n}^{\prime}(t)\right|\right)\right) \mathrm{d} t \leqslant \int_{a_{i}}^{b_{i}} \omega_{2}\left(\Delta\left(t-\xi_{n}\right)\right) \mathrm{d} t=\frac{1}{\Delta} \int_{\Delta\left(a_{i}-\xi_{n}\right)}^{\Delta\left(b_{i}-\xi_{n}\right)} \omega_{2}(t) \mathrm{d} t,
\end{aligned}
$$

and if $\left\{j_{0}\right\}=\mathbb{J} \backslash\left(\mathbb{J}_{n}^{1} \cup \mathbb{J}_{n}^{2}\right)$, then

$$
\begin{aligned}
\int_{a_{j_{0}}}^{b_{j_{0}}} \omega_{2}\left(g\left(\left|x_{n}^{\prime}(t)\right|\right)\right) \mathrm{d} t & \leqslant \int_{a_{j_{0}}}^{\xi_{n}} \omega_{2}\left(\Delta\left(\xi_{n}-t\right)\right) \mathrm{d} t+\int_{\xi_{n}}^{b_{j_{0}}} \omega_{2}\left(\Delta\left(t-\xi_{n}\right)\right) \mathrm{d} t \\
& =\frac{1}{\Delta}\left[\int_{0}^{\Delta\left(\xi_{n}-a_{j_{0}}\right)} \omega_{2}(t) \mathrm{d} t+\int_{0}^{\Delta\left(b_{j_{0}}-\xi_{n}\right)} \omega_{2}(t) \mathrm{d} t\right] .
\end{aligned}
$$

Since $\omega_{2} \in L_{1}([0, \Delta T])$ and

$$
\sum_{j \in \mathbb{J}_{n}^{1}} \Delta\left[\left(\xi_{n}-a_{j}\right)-\left(\xi_{n}-b_{j}\right)\right]+\sum_{i \in \mathbb{J}_{n}^{2}} \Delta\left[\left(b_{i}-\xi_{n}\right)-\left(a_{i}-\xi_{n}\right)\right]+E_{n}=\Delta \sum_{j \in \mathbb{J}}\left(b_{j}-a_{j}\right),
$$

we see from $(2.39)-(2.41)$ that $\left\{\omega_{2}\left(g\left(\left|x_{n}^{\prime}(t)\right|\right)\right)\right\}$ is UAC on $J$.

Finally, we consider the third sequence in (2.35). Let

$$
\Lambda=\min \left\{\frac{1}{4} c_{1}, \frac{1}{4}\left(T-c_{2}\right)\right\}
$$

and suppose that $b_{j}-a_{j} \leqslant \Lambda$ for each $j \in \mathbb{J}$. Set

$$
\begin{aligned}
\mathbb{I}_{1} & =\left\{j: j \in \mathbb{J}, a_{j} \leqslant \Lambda\right\}, \\
\mathbb{I}_{2} & =\left\{j: j \in \mathbb{J}, b_{j} \geqslant T-\Lambda\right\}, \\
\mathbb{X}_{n}^{1} & =\left\{j: j \in \mathbb{J} \backslash \mathbb{I}_{1},\left(a_{j}, b_{j}\right) \subset\left(0, \xi_{n}\right)\right\}, \\
\mathbb{X}_{n}^{2} & =\left\{j: j \in \mathbb{J} \backslash \mathbb{I}_{2},\left(a_{j}, b_{j}\right) \subset\left(\xi_{n}, T\right)\right\}
\end{aligned}
$$


for $n \in \mathbb{N}$. Then for each $j \in \mathbb{I}_{1}, i \in \mathbb{I}_{2}, k \in \mathbb{X}_{n}^{1}$ and $l \in \mathbb{X}_{n}^{2}$, we have (see (2.33) and $(2.34))$

$$
\begin{aligned}
\int_{a_{j}}^{b_{j}} h_{2}\left(x_{n}(t)\right) \omega_{2}\left(g\left(\left|x_{n}^{\prime}(t)\right|\right)\right) \mathrm{d} t & \leqslant \omega_{2}(2 \Lambda \Delta) \int_{a_{j}}^{b_{j}} h_{2}(\Delta t) \mathrm{d} t \\
= & \frac{\omega_{2}(2 \Lambda \Delta)}{\Delta} \int_{\Delta a_{j}}^{\Delta b_{j}} h_{2}(t) \mathrm{d} t \\
\int_{a_{i}}^{b_{i}} h_{2}\left(x_{n}(t)\right) \omega_{2}\left(g\left(\left|x_{n}^{\prime}(t)\right|\right)\right) \mathrm{d} t & \leqslant \omega_{2}(2 \Lambda \Delta) \int_{a_{i}}^{b_{i}} h_{2}(\Delta(T-t)) \mathrm{d} t \\
& =\frac{\omega_{2}(2 \Lambda \Delta)}{\Delta} \int_{\Delta\left(T-b_{i}\right)}^{\Delta\left(T-a_{i}\right)} h_{2}(t) \mathrm{d} t, \\
\int_{a_{k}}^{b_{k}} h_{2}\left(x_{n}(t)\right) \omega_{2}\left(g\left(\left|x_{n}^{\prime}(t)\right|\right)\right) \mathrm{d} t & \leqslant h_{2}(\Lambda \Delta) \int_{a_{k}}^{b_{k}} \omega_{2}\left(\Delta\left(\xi_{n}-t\right)\right) \mathrm{d} t \\
& =\frac{h_{2}(\Lambda \Delta)}{\Delta} \int_{\Delta\left(\xi_{n}-b_{k}\right)}^{\Delta\left(\xi_{n}-a_{k}\right)} \omega_{2}(t) \mathrm{d} t, \\
\int_{a_{l}}^{b_{l}} h_{2}\left(x_{n}(t)\right) \omega_{2}\left(g\left(\left|x_{n}^{\prime}(t)\right|\right)\right) \mathrm{d} t & \leqslant h_{2}(\Lambda \Delta) \int_{a_{l}}^{b_{l}} \omega_{2}\left(\Delta\left(t-\xi_{n}\right)\right) \mathrm{d} t \\
& =\frac{h_{2}(\Lambda \Delta)}{\Delta} \int_{\Delta\left(a_{l}-\xi_{n}\right)}^{\Delta\left(b_{l}-\xi_{n}\right)} \omega_{2}(t) \mathrm{d} t,
\end{aligned}
$$

and if $a_{k_{0}}<\xi_{n}<b_{k_{0}}$ for some $k_{0} \in \mathbb{J}$, then

$$
\begin{aligned}
\int_{a_{k_{0}}}^{b_{k_{0}}} h_{2}\left(x_{n}(t)\right) \omega_{2}\left(g\left(\left|x_{n}^{\prime}(t)\right|\right)\right) \mathrm{d} t \\
\qquad h_{2}(\Lambda \Delta)\left[\int_{a_{k_{0}}}^{\xi_{n}} \omega_{2}\left(\Delta\left(\xi_{n}-t\right)\right) \mathrm{d} t+\int_{\xi_{n}}^{b_{k_{0}}} \omega_{2}\left(\Delta\left(t-\xi_{n}\right)\right) \mathrm{d} t\right] \\
=\frac{h_{2}(\Lambda \Delta)}{\Delta}\left[\int_{0}^{\Delta\left(\xi_{n}-a_{k_{0}}\right)} \omega_{2}(t) \mathrm{d} t+\int_{0}^{\Delta\left(b_{k_{0}}-\xi_{n}\right)} \omega_{2}(t) \mathrm{d} t\right]
\end{aligned}
$$

Since

$$
\begin{aligned}
\sum_{j \in \mathbb{I}_{1}} \Delta\left(b_{j}-a_{j}\right)+\sum_{i \in \mathbb{I}_{2}} \Delta\left[\left(T-a_{i}\right)-\right. & \left.\left(T-b_{i}\right)\right]+\sum_{k \in \mathbb{X}_{n}^{1}} \Delta\left[\left(\xi_{n}-a_{k}\right)-\left(\xi_{n}-b_{k}\right)\right] \\
& +\sum_{l \in \mathbb{X}_{n}^{2}} \Delta\left[\left(b_{l}-\xi_{n}\right)-\left(a_{l}-\xi_{n}\right)\right]+H_{n}=\Delta \sum_{j \in \mathbb{J}}\left(b_{j}-a_{j}\right),
\end{aligned}
$$

where

$$
H_{n}= \begin{cases}0 & \text { if } \mathbb{J}=\mathbb{I}_{1} \cup \mathbb{I}_{2} \cup \mathbb{X}_{n}^{1} \cup \mathbb{X}_{n}^{2}, \\ \Delta\left(b_{k_{0}}-a_{k_{0}}\right) & \text { if }\left\{k_{0}\right\}=\mathbb{J} \backslash\left(\mathbb{I}_{1} \cup \mathbb{I}_{2} \cup \mathbb{X}_{n}^{1} \cup \mathbb{X}_{n}^{2}\right),\end{cases}
$$


it follows from (2.42)-(2.46) that

$$
\begin{aligned}
& \sum_{j \in \mathbb{J}} \int_{a_{j}}^{b_{j}} h_{2}\left(x_{n}(t)\right) \omega_{2}\left(g\left(\left|x_{n}^{\prime}(t)\right|\right)\right) \mathrm{d} t \\
& \quad \leqslant \frac{1}{\Delta} \max \left\{h_{2}(\Lambda \Delta), \omega_{2}(2 \Lambda \Delta)\right\}\left[\int_{\mathcal{M}_{n}^{1}}\left(h_{2}(t)+\omega_{2}(t)\right) \mathrm{d} t+\int_{\mathcal{M}_{n}^{2}}\left(h_{2}(t)+\omega_{2}(t)\right) \mathrm{d} t\right],
\end{aligned}
$$

where $\mathcal{M}_{n}^{i} \subset(0, \Delta T)$ are measurable sets, $\mu\left(\mathcal{M}_{n}^{i}\right) \leqslant \Delta \sum_{j \in \mathbb{J}}\left(b_{j}-a_{j}\right)$, for $i=1,2$ and each $n \in \mathbb{N}$. We know that $q_{2}, \omega_{2} \in L_{1}([0, \Delta T])$, and consequently (2.47) shows that the sequence $\left\{q_{2}\left(x_{n}(t)\right) \omega_{2}\left(g\left(\left|x_{n}^{\prime}(t)\right|\right)\right)\right\}$ is UAC on $J$.

\section{Existence results and an example}

Theorem 3.1. Let assumptions $\left(\mathrm{H}_{1}\right)-\left(\mathrm{H}_{3}\right)$ be satisfied. Then for each $A \in \mathbb{R}_{+}$, there exists a solution of $B V P(1.1)-(1.3)$.

Proof. Fix $A \in \mathbb{R}_{+}$. By Lemma 2.6, for each $n \in \mathbb{N}$, there exists a solution $x_{n}$ of BVP (2.3), (1.2), (1.3) with $\mu=\mu_{n}$ in (2.3). Consider the sequence $\left\{x_{n}\right\}$. Applying Lemmas 2.1-2.3 and 2.5 and arguing as in the proof of Lemma 2.6, there exist positive constants $P, \Delta, \mu_{A}$ and $0<c_{1}<c_{2}<T$ such that the inequalities (2.31)-(2.34) are satisfied for each $n \in \mathbb{N}$, where $\xi_{n} \in(0, T)$ denotes the unique zero of $x_{n}^{\prime}$. In addition, the sequence $\left\{f_{n}\left(t, x_{n}(t), x_{n}^{\prime}(t)\right)\right\}$ is UAC on $J$ by Lemma 2.6, which implies that $\left\{g\left(x_{n}^{\prime}(t)\right)\right\}$ is equicontinuous on $J$ and now the uniform continuity of $g^{-1}$ on $[-g(P), g(P)]$ and

$$
\left|x_{n}^{\prime}\left(t_{1}\right)-x_{n}^{\prime}\left(t_{2}\right)\right|=\left|g^{-1}\left(g\left(x_{n}^{\prime}\left(t_{1}\right)\right)\right)-g^{-1}\left(g\left(x_{n}^{\prime}\left(t_{2}\right)\right)\right)\right|, \quad t_{1}, t_{2} \in J, n \in \mathbb{N},
$$

show that $\left\{x_{n}^{\prime}(t)\right\}$ is equicontinuous on $J$. The Arzelà-Ascoli theorem and the BolzanoWeierstrass theorem, going if necessary to subsequences, imply that we can assume that $\left\{x_{n}\right\}$ is convergent in $C^{1}(J ; \mathbb{R})$ and $\left\{\mu_{n}\right\}$ and $\left\{\xi_{n}\right\}$ are convergent in $\mathbb{R}$. Let $\lim _{n \rightarrow \infty} x_{n}=x, \lim _{n \rightarrow \infty} \mu_{n}=\mu$ and $\lim _{n \rightarrow \infty} \xi_{n}=\xi$. Then $x \in C^{1}(J ; \mathbb{R}), x(0)=x(T)=$ $0, \max \{x(t): t \in J\}=A$ and

$$
c_{1} \leqslant \xi \leqslant c_{2}, \quad \mu_{A} \leqslant \mu \leqslant \frac{1}{a}\left(A\left(1+\frac{1}{\beta}\right)\right)^{\beta}\left(\frac{2}{T}\right)^{1+\beta} .
$$

Taking the limit as $n \rightarrow \infty$ in (2.33) and (2.34), we get

$$
x(t) \geqslant \begin{cases}\Delta t & \text { for } t \in[0, \xi], \\ \Delta(T-t) & \text { for } t \in(\xi, T]\end{cases}
$$

and

$$
\begin{aligned}
g\left(x^{\prime}(t)\right) \geqslant \Delta(\xi-t) & \text { for } t \in[0, \xi], \\
g\left(\left|x^{\prime}(t)\right|\right) \geqslant \Delta(t-\xi) & \text { for } t \in(\xi, T] .
\end{aligned}
$$


It follows from the construction of the functions $f_{n} \in \operatorname{Car}\left(J \times \mathbb{R}^{2} ; \mathbb{R}\right)$ that there exists $\mathcal{V} \subset J, \mu(\mathcal{V})=0$, such that $f_{n}(t, \cdot, \cdot)$ is continuous on $\mathbb{R}^{2}$ for $t \in J \backslash \mathcal{V}$ and $n \in \mathbb{N}$. Then

$$
\lim _{n \rightarrow \infty} f_{n}\left(t, x_{n}(t), x_{n}^{\prime}(t)\right)=f\left(t, x(t), x^{\prime}(t)\right) \quad \text { for } t \in J \backslash(\mathcal{V} \cup\{0, \xi, T\}) .
$$

Consequently, $f\left(t, x(t), x^{\prime}(t)\right) \in L_{1}(J)$ and

$$
\lim _{n \rightarrow \infty} \int_{0}^{t} f_{n}\left(s, x_{n}(s), x_{n}^{\prime}(s)\right) \mathrm{d} s=\int_{0}^{t} f\left(s, x(s), x^{\prime}(s)\right) \mathrm{d} s \quad \text { for } t \in J
$$

by Vitali's convergence theorem. Finally, letting $n \rightarrow \infty$ in the equalities

$$
g\left(x_{n}^{\prime}(t)\right)=g\left(x_{n}^{\prime}(0)\right)+\mu_{n} \int_{0}^{t} f_{n}\left(s, x_{n}(s), x_{n}^{\prime}(s)\right) \mathrm{d} s, \quad t \in J, n \in \mathbb{N},
$$

we have

$$
g\left(x^{\prime}(t)\right)=g\left(x^{\prime}(0)\right)+\mu \int_{0}^{t} f\left(s, x(s), x^{\prime}(s)\right) \mathrm{d} s, \quad t \in J .
$$

Hence $g\left(x^{\prime}\right) \in \mathrm{AC}(J ; \mathbb{R})$ and $x$ is a solution of (1.1). We have proved that $x$ is a solution of BVP (1.1)-(1.3).

For the continuous function $f$ in (1.1) the following corollary immediately follows from Theorem 3.1 and the above considerations.

Corollary 3.2. Let $f \in C^{0}\left(J \times \mathbb{R}_{+} \times \mathbb{R}_{0} ; \mathbb{R}\right)$ and assume that $\left(\mathrm{H}_{1}\right)-\left(\mathrm{H}_{3}\right)$ are satisfied. Then for each $A \in \mathbb{R}_{+}$, there exists a solution $x$ of $B V P$ (1.1)-(1.3). If $\xi \in(0, T)$ denotes the (unique) zero of $x^{\prime}$, then $g\left(x^{\prime}\right) \in C^{1}(J \backslash\{0, \xi, T\} ; \mathbb{R})$ and (1.1) is satisfied for $t \in J \backslash\{0, \xi, T\}$.

Example 3.3. Consider the differential equation

$$
\left(\left|x^{\prime}\right|^{p} \operatorname{sgn} x^{\prime}\right)^{\prime}+\mu\left(q_{1}(t)+q_{2}(t) x^{\gamma_{1}}+\frac{q_{3}(t)}{x^{\gamma_{2}}}+\frac{q_{4}(t)}{x^{\gamma_{3}}\left|x^{\prime}\right|^{\eta_{1}}}+\frac{q_{5}(t)}{\left|x^{\prime}\right|^{\eta_{2}}}+q_{6}(t)\left|x^{\prime}\right|^{\eta_{3}}\right)=0,
$$

where $p \in \mathbb{R}_{+}, q_{1} \in L_{1}(J ;[a, \infty))$ with an $a \in \mathbb{R}_{+}, q_{j} \in L_{\infty}(J ;[0, \infty))(2 \leqslant j \leqslant 6)$, $\gamma_{1} \in[0, \infty), \gamma_{2}, \gamma_{3} \in(0,1), \eta_{1}, \eta_{2} \in(0, p)$ and $\eta_{3} \in(0,1+p)$. Equation (3.1) is the special case of (1.1) with

$$
g(u)=|u|^{p} \operatorname{sgn} u, \quad u \in \mathbb{R},
$$

and

$$
f(t, u, v)=-\left(q_{1}(t)+q_{2}(t) u^{\gamma_{1}}+\frac{q_{3}(t)}{u^{\gamma_{2}}}+\frac{q_{4}(t)}{u^{\gamma_{3}}|v|^{\eta_{1}}}+\frac{q_{5}(t)}{|v|^{\eta_{2}}}+q_{6}(t)|v|^{\eta_{3}}\right)
$$

for $(t, u, v) \in J \times \mathbb{R}_{+} \times \mathbb{R}_{0}$. Let $M=\max \left\{\left\|q_{j}\right\|_{\infty}: 1 \leqslant j \leqslant 6\right\}$. Then

$$
a \leqslant-f(t, u, v) \leqslant 2 M\left[h_{1}(u)+h_{2}(u)\right]\left[\omega_{1}(g(|v|))+\omega_{2}(g(|v|))\right]
$$

for a.e. $t \in J$ and each $(u, v) \in \mathbb{R}_{+} \times \mathbb{R}_{0}$, where

$$
h_{1}(u)=1+u^{\gamma_{1}}, \quad h_{2}(u)=\frac{1}{u^{\gamma_{2}}}+\frac{1}{u^{\gamma_{3}}}, \quad \omega_{1}(u)=1+u^{\eta_{3 / p}}, \quad \omega_{2}(u)=1+\frac{1}{u^{\max \left\{\eta_{1}, \eta_{2}\right\} / p}} .
$$


Hence $g$ satisfies assumption $\left(\mathrm{H}_{1}\right)$ with $\beta=p$ and $f$ satisfies assumptions $\left(\mathrm{H}_{2}\right)$ and $\left(\mathrm{H}_{3}\right)$ with $\varphi(t)=2 M$. By Theorem 3.1, for each $A \in \mathbb{R}_{+}$, there exists a solution of BVP (3.1), (1.2), (1.3).

Acknowledgements. S.S. was supported by grant no. 201/01/1451 of the Grant Agency of the Czech Republic and by the Council of the Czech Government J14/98: 153100011.

\section{References}

1. R. P. Agarwal and D. O'Regan, Singular boundary value problems for superlinear second order ordinary and delay differential equations, J. Diff. Eqns 130 (1996), 333-355.

2. R. P. Agarwal and D. O'Regan, Positive solutions to superlinear singular boundary value problems, J. Computat. Appl. Math. 88 (1998), 129-147.

3. R. P. Agarwal, D. O'Regan and S. StaněK, Singular Lidstone boundary value problem with given maximal values for solutions, Nonlin. Analysis 55 (2003), 859-881.

4. R. P. Agarwal, D. O'REgan, I. RachO̊nková And S. StAnĚK, Two-point higher order BVPs with singularities in phase variables, Comput. Math. Appl. 46 (2003), 1799-1826.

5. R. G. BARTLE, A modern theory of integration (American Mathematical Society, Providence, RI, 2001).

6. A. Boucherif, Nonlinear multipoint boundary value problem, Nonlin. Analysis 10 (1986), 957-964.

7. K. Deimling, Nonlinear functional analysis (Springer, 1985).

8. J. A. Gatica, V. Oliker and P. Waltman, Singular nonlinear boundary value problems for second order ordinary differential equations, J. Diff. Eqns 79 (1989), 62-78.

9. M. Greguš, F. Neuman and F. M. Arscott, Three-point boundary value problems in differential equations, J. Lond. Math. Soc. 3 (1971), 429-436.

10. E. Hewitt And S. Stromberg, Real and abstract analysis (Springer, 1965).

11. T. JANKOWski, Monotone iterations for first order differential equations with a parameter, Acta Math. Hungar. 84 (1999), 65-80.

12. I. T. Kiguradze And B. L. SheKhter, Singular boundary value problems for second order ordinary differential equations, in Current problems in mathematics: newest results, vol. 30, pp. 105-201 (Moscow, 1987) (in Russian) (English transl.: J. Sov. Math. 43 (1988), 2340-2417).

13. N. S. Kurpel and A. G. Marusjak, On the multipoint boundary value problem for differential equations with parameters, Ukrain. Math. Z. 32 (1980), 223-226 (in Russian).

14. D. O'REgAn, Theory of singular boundary value problems (World Scientific, 1994).

15. D. O'REGAN, Existence principles and theory for singular Dirichlet boundary value problems, Diff. Eqns Dynam. Syst. 3 (1995), 289-304.

16. I. RAChO̊nková And S. StanĚK, Sign-changing solutions of singular Dirichlet boundary value problems, J. Inequal. Applic., in press.

17. M. Ronto, On numerical-analytic method for BVPs with parameters, Publ. Univ. Miskolc D 36 (1996), 125-132.

18. S. StAnĚK, On a class of five-point boundary value problems in second-order functional differential equations with parameter, Acta Math. Hungar. 62 (1993), 253-262.

19. S. STANĚK, On a class of functional boundary value problems for the equation $x^{\prime \prime}=$ $f\left(t, x, x^{\prime}, x^{\prime \prime}, \lambda\right)$, Ann. Polon. Math. 59 (1994), 225-237.

20. S. STANĚK, On a class of functional boundary value problems for nonlinear third-order functional differential equations depending on the parameter, Arch. Math. 62 (1994), 462-469. 
21. S. STANĚK, On functional boundary value problems for systems of functional differential equations depending on parameters, Arch. Math. 64 (1995), 434-443.

22. S. StAnĚK, Positive solutions of singular positone Dirichlet boundary value problems, Math. Comput. Model. 33 (2001), 341-351.

23. S. StAnĚK, Positive solutions of singular Dirichlet and periodic boundary value problems, Comput. Math. Appl. 43 (2002), 681-692.

24. S. D. TAliAferro, A nonlinear singular boundary value problem, Nonlin. Analysis 3 (1979), 897-904.

25. A. Tineo, Existence theorems for a singular two-point Dirichlet problem, Nonlin. Analysis 19 (1992), 323-333.

26. J. WANG, Solvability of singular nonlinear two-point boundary value problems, Nonlin. Analysis 24 (1995), 555-561.

27. Z. ZENGQUin, Uniqueness of positive solutions for singular nonlinear second-order boundary-value problems, Nonlin. Analysis 23 (1994), 755-765. 